\title{
Um olhar sobre o diabetes na infância e na juventude: nem todos são Tipo 1
}

\author{
A look on diabetes in children and youth: not all are type 1
}

Mauren Isfer ANGHEBEM-OLIVEIRA

Escola de Saúde e Biociências. Pontificia Universidade Catótlica do Paraná. Rua Imaculada Conceição, 1155 Bairro Prado Velho, Curitiba, PR. CEP: 80215-901.

E-mail: mauren_isfer@hotmail.com

\section{ABSTRACT}

Diabetes mellitus (DM) is characterized by chronic hyperglycemia, which causes endothelial dysfunction and vascular complications, such as retinopathy, nephropathy and acute myocardial infarction. The progress in understanding the pathophysiology and clinical characteristics of diabetes, its classification has been updated since the correct classification of diabetes impacts on prognosis and treatment of the patient. Currently, diabetes is classified as type 1 diabetes, type 2 diabetes, gestational DM and other specific types that include MODY (Maturity Onset Diabetes of the Young). The aim of this review is to show that not all diabetes diagnosed in childhood and youth is type $1 \mathrm{DM}$. The correct diagnosis and classification of diabetes are primordial, since the prognosis and treatment may differ depending on the cause which predisposed the child or juvenile to disease.

Keywords: diabetes, type 1 diabetes, MODY

\section{RESUMO}

O Diabetes mellitus (DM) é caracterizado por um quadro de hiperglicemia crônica, que com os anos pode causar disfunção endotelial e sérias complicações vasculares, como a retinopatia, nefropatia e o infarto agudo do miocárdio. À medida que a ciência avança na compreensão da fisiopatologia e das características clínico-laboratoriais do diabetes, sua classificação tem sido adaptada, justamente porque a correta classificação do diabetes impacta no prognóstico e tratamento do paciente. Atualmente, o diabetes é classificado em DM tipo 1, DM tipo 2, DM gestacional e Outros Tipos Específicos, que inclui a categoria MODY (do inglês, Maturity Onset Diabetes of the Young) ou diabetes da maturidade com início na juventude. O que esta revisão pretende mostrar é quem nem todo diabetes diagnosticado na infância e na juventude é DM tipo 1 . O correto diagnóstico e classificação do DM são fundamentais, uma vez que o prognóstico e o tratamento podem diferir dependendo da causa que predispôs a criança ou adolescente à doença.

Palavras Chave: diabetes, diabetes tipo 1, MODY

\section{INTRODUÇÃO}

O Diabetes mellitus (DM) é uma doença crônica caracterizada por hiperglicemia resultante de defeito na ação e/ou secreção da insulina. Ao longo dos anos a hiperglicemia crônica promove disfunção endotelial, processo envolvido nas complicações micro e macrovasculares do diabetes (1).

Há alguns anos, o DM era classificado como Diabetes mellitus tipo 1 (DM1) ou insulino-dependente e Diabetes mellitus tipo 2 (DM2) ou insulino-independente. Essa classificação se baseava nos sinais e sintomas mais característicos a cada uma das formas. Entretanto, os termos insulino-dependente e insulino-independente utilizados para caracterizar o DM1 e o DM2 não são mais utilizados, uma vez que indivíduos com DM2 necessitarão da administração de insulina quando o uso de hipoglicemiantes orais não atingir a meta terapêutica (2).

$\mathrm{O}$ crescente entendimento em relação à fisiopatologia do diabetes nas últimas décadas possibilitou a individualização das síndromes hiperglicêmicas, classificando outros tipos específicos de diabetes, caracterizados por mutações genéticas das células $\beta$ pancreáticas e ação da insulina, doenças que acometem o pâncreas, infecções, diabetes relacionado ao uso de medicamentos, endocrinopatias e outras condições, conforme listado na Tabela 1 (3). 
Esta classificação determina 4 grupos: 1) DM1, onde há destruição auto-imune das células beta pancreáticas resultando na ausência de insulina; 2) DM2, onde ocorre resistência relativa à insulina; 3) Outros tipos específicos, onde várias condições podem causar hiperglicemia, caracterizando o diabetes; e, 4) Diabetes gestacional, aquele diagnosticado durante o período da gestação, e que ocorre pela elevação dos hormônios contra-reguladores da insulina, pelo desequilíbrio fisiológico imposto pela gravidez e seus fatores genéticos e/ ou ambientais (4).

$\mathrm{O}$ aumento mundial de novos casos de diabetes sugere uma pandemia emergente (5). No Brasil, segundo dados do Ministério da Saúde, em 2010 o número de internações em virtude das complicações do diabetes passou de 148.000 , com custos superiores a 80 milhões de reais. Esses dados indicam a necessidade de diagnóstico e tratamento precoce de indivíduos com DM, independente da idade, a fim de minimizar as complicações causadas pela hiperglicemia crônica, as quais causam um impacto direto no número de internações e nos gastos do Sistema Único de Saúde (6).

\section{DIABETES MELLITUS NA INFÂNCIA E NA JUVENTUDE}

No momento do diagnóstico, é interessante que seja feita a correta classificação do DM, uma vez que as implicações clínicas, prognóstico e tratamento podem diferir dependendo da causa que predispôs a criança ou adolescente à doença (7). O DM1 é o tipo mais comum em crianças e adolescentes, com uma frequência de aproximadamente $95 \%$, mas não é o único que pode acometer esse grupo de indivíduos. A frequência de DM2 em jovens e adolescentes, anteriormente considerado como o diabetes do idoso, tem representado uma parcela significativa dos novos casos diagnosticados
(8). Em alguns países africanos e asiáticos a incidência de DM2 entre jovens é superior à de DM1, assim como entre jovens hispano-americanos $(9 ; 10)$. A similaridade da apresentação desses dois tipos de DM em crianças e adolescentes, reflexo de fatores ambientais como sedentarismo e obesidade, dificulta a sua classificação (11). Não obstante, não é incomum uma criança receber o diagnóstico de DM1 ou DM2, quando, de fato, é portadora de diabetes monogênico. Em crianças e adolescentes cuja apresentação clínica de DM não se enquadra bem no tipo 1 tampouco no tipo 2 , devem ser consideradas outras formas de diabetes, como as de causa monogênica (12).

A suspeita inicial de diabetes monogênico se baseia nas peculiaridades clínicas de cada subtipo, mas o diagnóstico definitivo depende de estudos genéticos. A definição da forma monogênica de diabetes favorece um prognóstico mais preciso e, consequentemente, um tratamento mais adequado (7). A Tabela 2 sumariza algumas diferenças entre os principais tipos de diabetes na infância e juventude.

MODY (do inglês, $\underline{\text { Maturity } \underline{\text { Onset }} \text { Diabetes of }}$ the Young) ou diabetes da maturidade com início na juventude é uma forma monogênica de DM, associada a defeitos genéticos na função da célula beta de secretar insulina (13). Pacientes com MODY frequentemente apresentam uma ou mais das seguintes características: 1) histórico familiar ou diabetes de qualquer tipo; 2) não são dependentes de insulina; 3) ausência de positividade para anticorpos anti-ilhotas pancreáticas e, 4) sinais de produção endógena de insulina evidenciados pela detecção de peptídeo-C dosado na presença de hiperglicemia, necessidade de baixas doses de insulina ou ausência de cetoacidose quando a insulina é omitida. Esse quadro não é típico do DM1, o que sugere o caráter monogênico da doença (14). 
Tabela 1: Classificação etiológica da Diabetes Mellitus (4)

\begin{tabular}{|c|c|c|}
\hline Tipo de Diabetes & Etiologia & Frequência \\
\hline Diabetes melittus tipo 1 & $\begin{array}{l}\text { Destruição das células beta-pancreáticas, gerando deficiência absoluta de insulina. Esta destru- } \\
\text { ição pode ser imunomediada }(70 \%) \text { ou de causa idiopática }(30 \%) \text {. }\end{array}$ & $5-10 \%$ \\
\hline Diabetes mellitus tipo 2 & Defeito na ação e/ou secreção da insulina, com resistência à insulina. & $90-95 \%$ \\
\hline \multirow[t]{8}{*}{ Outros tipos específicos } & $\begin{array}{l}\text { A. Defeitos genéticos na função da célula beta } \\
\text { 1. MODY } 1 \text { - defeito no gene HNF- } 4 \alpha \text {, cromossomo } 20 \\
\text { 2. MODY } 2 \text { - defeito no gene da glicoquinase, cromossomo } 7 \\
\text { 3. MODY } 3 \text { - defeito no gene HNF-1 } \alpha \text {, cromossomo } 12 \\
\text { 4. MODY } 4 \text { - defeito no gene IPF-1 (insulin promoter factor-1), cromossomo } 13 \\
\text { 5. MODY } 5 \text { - defeito no gene HNF-1 } \beta \text {, cromossomo } 17 \\
\text { 6. MODY } 6 \text { - defeito no gene NeuroD1, cromossomo } 2 \\
\text { 7. DM mitocondrial - defeito no DNA mitocondrial } \\
\text { 8. Outros }\end{array}$ & \multirow[t]{8}{*}{ Rara } \\
\hline & $\begin{array}{l}\text { B. Defeitos genéticos na ação da insulina } \\
\text { 1. Resistência à insulina do tipo A } \\
\text { 2. Leprechaunismo } \\
\text { 3. Síndrome de Rabson-Mendenhall } \\
\text { 4. Diabetes lipoatrófico } \\
\text { 5. Outros }\end{array}$ & \\
\hline & $\begin{array}{l}\text { C. Doenças do pâncreas exócrino } \\
\text { 1. Pancreatite } \\
\text { 2. Trauma ou pancreatectomia } \\
\text { 3. Neoplasia } \\
\text { 4. Fibrose cística } \\
\text { 5. Hemocromatose } \\
\text { 6. Pancreatopatia fibrocalculosa } \\
\text { 7. Outros }\end{array}$ & \\
\hline & $\begin{array}{l}\text { D. Endocrinopatias } \\
\text { 1. Acromegalia } \\
\text { 2. Síndrome de Cushing } \\
\text { 3. Glucagonoma } \\
\text { 4. Feocromocitoma } \\
\text { 5. Hipertireoidismo } \\
\text { 6. Somatostatinoma } \\
\text { 7. Aldosteronoma } \\
\text { 8. Outros }\end{array}$ & \\
\hline & $\begin{array}{l}\text { E. Induzido por drogas ou agentes químicos } \\
\text { 1. Algumas toxinas } \\
\text { 2. Pentamidina } \\
\text { 3. Ácido nicotínico } \\
\text { 4. Glicocorticóides } \\
\text { 5. Hormônios tireoideanos } \\
\text { 6. Diazóxido } \\
\text { 7. Agonistas beta-adrenérgicos } \\
\text { 8. Tiazídicos } \\
\text { 9. Fenitoína } \\
\text { 10. Interferon } \\
\text { 11. Outros }\end{array}$ & \\
\hline & $\begin{array}{l}\text { F. Infecções } \\
\text { 1. Rubéola congênita } \\
\text { 2. Citomegalovírus } \\
\text { 3. Outros }\end{array}$ & \\
\hline & $\begin{array}{l}\text { G. Formas incomuns de diabetes auto-imune } \\
\text { 1. Sindrome "Stiff-man" } \\
\text { 2. Anticorpos anti-receptores de insulina } \\
\text { 3. Outros }\end{array}$ & \\
\hline & $\begin{array}{l}\text { H. Outras síndromes genéticas por vezes associadas ao diabetes } \\
\text { 1. Síndrome de Down } \\
\text { 2. Síndrome Klinefelter } \\
\text { 3. Síndrome de Turner } \\
\text { 4. Síndrome de Wolfram } \\
\text { 5. Ataxia de Friedreich } \\
\text { 6. Coreia de Huntington } \\
\text { 7. Síndrome de Laurence-Moon-Biedl } \\
\text { 8. Distrofia miotônica } \\
\text { 9. Porfiria } \\
\text { 10. Síndrome de Prader-Willi } \\
\text { 11. Outros }\end{array}$ & \\
\hline Diabetes gestacional & Intolerância à glicose, de grau variável, com início ou diagnóstico no período da gestação. & $7 \%$ de todas as gestações * \\
\hline
\end{tabular}

* Podendo variar de 1 a 14\%, dependendo da população estudada.

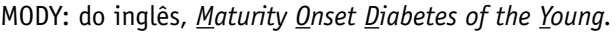


Da mesma forma, em jovens aparentemente portadores de DM2, a ausência de resistência à insulina, caracterizada pela falta de obesidade, valores de triglicérides normais e de HDL-C normais ou aumentados, e a ausência de manifestações cutâneas como acanthosis nigricans podem indicar a presença de MODY $(14 ; 15 ; 16)$.

Um grande estudo envolvendo crianças e adolescentes diabéticos comparou as características clínicas daqueles com MODY e com DM2, e mostrou que todos os subtipos de MODY podem apresentar fatores de risco macrovascular, como hipertensão e dislipidemia. No entanto, embora presente nos indivíduos com MODY, esses fatores são mais frequentes nas crianças com DM2 (17).

\section{UM DESTAQUE PARA O DIABETES TIPO MODY}

Em 1960, o pesquisador americano Stefan S. Fajans foi o primeiro a descrever um grupo de crianças não obesas com histórico familiar de DM2, que apresentavam sintomas leves de DM e mostravam bom controle metabólico com o uso do hipoglicemiante sulfoniluréia. Mais tarde utilizava-se pela primeira vez o termo MODY para indivíduos com estas características, destacando-se a herança familiar autossômica dominante nesta forma de diabetes, que o diferenciava do DM1 (18). O diabetes MODY representa de 3-5\% dos casos de pacientes diagnosticados com DM2, com diagnóstico de hiperglicemia antes dos 25 anos $(19 ; 20)$.

Seis subtipos de MODY foram associados com mutações genéticas bem definidas e caracterizadas, embora já tenham sido reportados outros subtipos. Uma porcentagem significativa de indivíduos com fenótipo clássico de MODY não apresenta mutações em nenhum dos seis genes conhecidos (21).

Com exceção do MODY 2, cuja mutação é no gene da glicoquinase e desencadeia uma hiperglicemia leve, as demais formas de MODY apresentam mutações secundárias em fatores de transcrição expressos nas células beta-pancreáticas, com quadros de hiperglicemia severa $(22 ; 23 ; 24)$. A apresentação clínica dos indivíduos com MODY é heterogênea, variando conforme a etiologia genética (Tabela 3 ).

A verdadeira prevalência de MODY é incerta para a maioria das populações ( 1 a $5 \%$ ), incluindo a brasileira, pela dificuldade em diferenciar os tipos de DM, principalmente DM2 e MODY $(25,26)$.

Tabela 2. Características do Diabetes Melitus Tipo 1 (DM1),

Diabetes Melitus Tipo 2 (DM2) e Diabetes monogênico (7;10).

\begin{tabular}{|c|c|c|c|}
\hline Caracteristicas & $\begin{array}{l}\text { Diabetes mellitus tipo } 1 \\
\text { (DM1) }\end{array}$ & Diabetes mellitus tipo 2 (DM2) & $\begin{array}{l}\text { Diabetes } \\
\text { monogênico }\end{array}$ \\
\hline Predisposição genética & Poligênica & Poligênica & Autossômica dominante \\
\hline Histórico familiar & $<15 \%$ & $>50 \%$ & $100 \%$ \\
\hline Idade da apresentação & $\begin{array}{l}\text { Diagnosticado } 50 \% \text { durante a infância, } \\
50 \% \text { na idade adulta, } 99 \% \text { em crianças } \\
\text { diabéticas com }<10 \text { anos. }\end{array}$ & $\begin{array}{l}\text { Diagnosticado }>95 \% \text { em adultos, e de } 10- \\
15 \% \text { em adolescentes. }\end{array}$ & $\begin{array}{l}\text { Diagnosticado, normal- } \\
\text { mente, antes dos } 25 \text { anos. }\end{array}$ \\
\hline Início & Agudo & Variável: de agudo a insidioso & $\begin{array}{l}\text { Variável: de agudo a in- } \\
\text { sidioso }\end{array}$ \\
\hline Autoimunidade & Positiva $(90-95 \%)$ & Negativa & Negativa \\
\hline Anticorpos anti-ilhotas & Sim ( $100 \%$ dos casos $)$ & $\begin{array}{l}\text { Não, mas não são incomuns resultados fal- } \\
\text { so-positivos. }\end{array}$ & $\begin{array}{l}\text { Não, mas pacientes MODY } \\
\text { podem desenvolver em. }\end{array}$ \\
\hline Obesidade & Rara & Frequente $(>90 \%)$ & Rara \\
\hline Cetose & Frequente & Rara & Rara \\
\hline Acanthosis nigricans* & Ausente & Presente & Ausente \\
\hline Dependência à insulina & $\begin{array}{l}\text { Completa, na maioria dos casos. } \\
\text { Algumas vezes, desenvolve lentamente. }\end{array}$ & Inicialmente ausente, na maioria dos casos. & $\begin{array}{l}\text { Inicialmente ausente, na } \\
\text { maioria dos casos. }\end{array}$ \\
\hline
\end{tabular}

MODY: Maturity Onset Diabetes of the Young.

* Condição dermatológica caracterizada por espessamento, hiperpigmentação e acentuação das linhas da pele,

associada a distúrbios metabólicos, como o DM. 
Embora algumas características fenotípicas possam direcionar para subtipos específicos de MODY, o diagnóstico é basicamente molecular. Esse fato torna-se um obstáculo pelo alto custo das análises genéticas e baixa acessibilidade para muitos pacientes. Gloyn e cols. (2012) falharam na busca de ferramentas diagnósticas mais acessíveis, como biomarcadores urinários que pudessem diferenciar os tipos de diabetes em crianças e jovens (27). Já a determinação sérica da proteína-C-reativa ultra-sensível tem se mostrado promissora como biomarcador do MODY 3, pois apresenta valores significativamente reduzidos em portadores da mutação no gene HNF-1 $\alpha$ (26), assim como a apolipoproteína M, que parece ser útil na diferenciação entre MODY 3 e DM1, já que suas concentrações são menores nos indivíduos com MODY 3 (28).

\section{MODY 1}

O MODY 1 é caracterizado pela deficiência na secreção de insulina estimulada pela glicose (29). A mutação heterozigótica no gene do fator de transcrição nuclear do hepatócito - $4 \alpha$ (HNF-4 $\alpha$ ), localizado no braço longo do cromossomo 20 (20q13), é considerado uma forma rara do MODY (30).

O HNF-4 $\alpha$ faz parte de um conjunto de fatores que regulam a expressão de diversos genes no fígado e pâncreas. O HNF-4 $\alpha$ participa da regulação da produção e secreção de insulina em resposta à glicose. O HNF-4 $\alpha$ é regulado por dois promotores: promotor fígado-específico P1 e o promotor P2, o qual regula a transcrição nas células $\beta$ pancreáticas (31).

Indivíduos portadores da mutação no gene HNF- $4 \alpha$ desenvolvem o diabetes por volta dos 25 anos, por perda progressiva da função da célula beta, ainda não bem determinada. A detecção de MODY 1 entre indivíduos com DM2 pode se tornar difícil, no entanto, histórico familiar de diabetes antes dos 40 anos e a ausência de marcadores de resistência à insulina ou obesidade em jovens com diagnóstico de DM2 são dados clínicos relevantes para o diagnóstico (32).

\section{MODY 2}

No subtipo 2 ocorre uma mutação secundária no gene codificador da enzima glicoquinase (GCK), localizada no cromossomo $7 \mathrm{p} 15-1-7 \mathrm{p} 15-3$ (31). A GCK catalisa a reação de fosforilação da glicose. A utilização da glicose aumenta a produção de ATP resultando em bloqueio de canais de potássio ATP-dependentes. O bloqueio desses canais induz uma despolarização da membrana e abertura de canais de cálcio, e o influxo desses íons, causando a mobilização de vesículas secretoras com insulina. Mutações no gene da GCK resultam em fluxo glicolótico na célula beta reduzido, com secreção defeituosa de insulina (33). O MODY 2 é a causa mais frequentes de diabetes monogênico em crianças assintomáticas com hiperglicemia suave persistente ou glicosúria $(21,22)$.

Diferente do fenótipo do MODY 1 e MODY 3, o MODY 2 apresenta uma forma mais branda de diabetes, muitas vezes apenas com quadros de intolerância a glicose. Normalmente é assintomático e é diagnosticado durante o exame clínico-laboratorial em crianças e gestantes (34). O MODY 2 tem melhor prognóstico que os demais, e, na maioria dos casos, o tratamento farmacológico não é necessário já que a insulina ou hipoglicemiantes orais não melhoram o controle glicêmico $(35 ; 36)$.

\section{MODY 3}

O subtipo 3 é caracterizado por uma mutação no gene do fator nuclear do hepatócito $-1 \alpha$ (HNF$1 \alpha$ ) que está localizado no braço longo do cromossomo 12 (12q24) e codifica proteínas nucleares expressas no fígado, rins, células $\beta$ pancreáticas e em alguns outros tecidos (37) 
Tabela 3. Principais características clínicas e genéticas dos subtipos de MODY (12).

\begin{tabular}{|c|c|c|c|c|c|}
\hline & 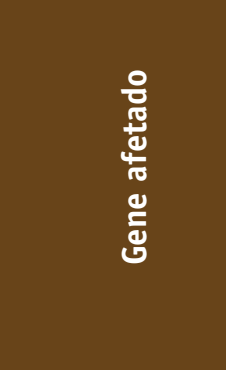 & 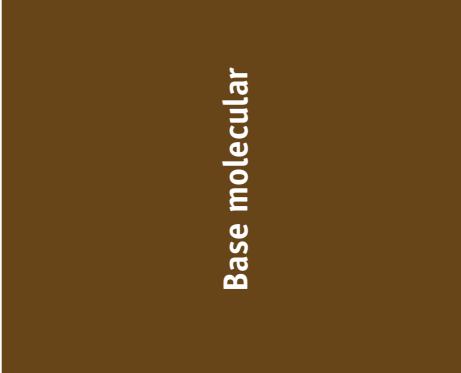 & •를 & 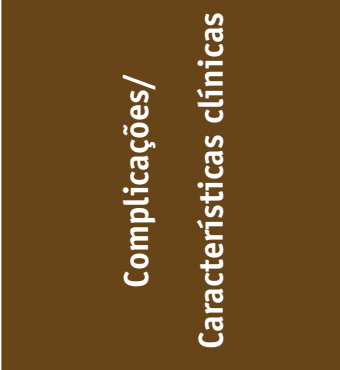 & 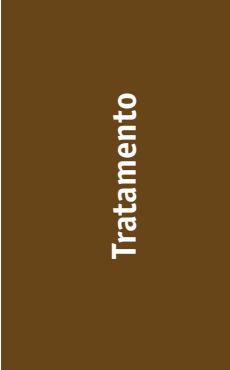 \\
\hline MODY 1 & $\begin{array}{l}\mathrm{HNF}-4 \alpha \\
(20 q 13)\end{array}$ & $\begin{array}{l}\text { Defeito na transcrição gênica nas } \\
\text { células } \beta \text {, causando defeitos na si- } \\
\text { nalização metabólica da secreção } \\
\text { da insulina }\end{array}$ & $5 \%$ & $\begin{array}{l}\text { IGT progressiva; compli- } \\
\text { cações microvasculares; } \\
\text { HDL-colesterol baixo }\end{array}$ & Sulfoniluréia \\
\hline MODY 2 & $\begin{array}{l}\text { Glucoquinase } \\
\text { (7p15.1-7p15.3) }\end{array}$ & $\begin{array}{l}\text { Defeito na sensibilidade das célu- } \\
\text { las } \beta \text { para glicose devido à redução } \\
\text { de sua fosforilação; defeito na es- } \\
\text { tocagem hepática de glicose como } \\
\text { glicogênio }\end{array}$ & $15 \%$ & $\begin{array}{l}\text { Hiperglicemia leve e es- } \\
\text { tável; complicações mi- } \\
\text { crovasculares são raras }\end{array}$ & $\begin{array}{l}\text { Dieta e exercícios } \\
\text { físicos }\end{array}$ \\
\hline MODY 3 & $\begin{array}{l}\text { HNF-1 } 1 \alpha \\
(12 q 24)\end{array}$ & $\begin{array}{l}\text { Defeito na transcrição gênica nas } \\
\text { células beta, causando defeitos na } \\
\text { sinalização metabólica da secreção } \\
\text { da insulina }\end{array}$ & $70 \%$ & $\begin{array}{l}\text { IGT progressiva; compli- } \\
\text { cações microvasculares }\end{array}$ & Sulfoniluréia \\
\hline MODY 4 & $\begin{array}{l}\text { IPF-1 } \\
(13 q 12)\end{array}$ & $\begin{array}{l}\text { Defeito na transcrição da regula- } \\
\text { ção/desenvolvimento das células } \beta\end{array}$ & $<1 \%$ & IGT progressiva & Sulfoniluréia \\
\hline MODY 5 & $\begin{array}{l}\text { HNF-1 } \beta \\
\text { (17cen-q21) }\end{array}$ & $\begin{array}{l}\text { Defeito na transcrição gênica nas } \\
\text { células } \beta \text {, causando defeitos na si- } \\
\text { nalização metabólica da secreção } \\
\text { da insulina }\end{array}$ & $2 \%$ & $\begin{array}{l}\text { IGT progressiva; compli- } \\
\text { cações microvasculares; } \\
\text { anormalidades renais e } \\
\text { atrofia pancreática; }\end{array}$ & Insulina \\
\hline MODY 6 & $\begin{array}{l}\text { NeuroD1/ Beta2 } \\
(2 q 32)\end{array}$ & $\begin{array}{l}\text { Defeito na transcrição da regula- } \\
\text { ção/desenvolvimento das células } \beta\end{array}$ & Rara & IGT progressiva & Insulina \\
\hline
\end{tabular}

IGT: Intolerância oral à glicose

O HNF-1 $\alpha$ parece ter papel fundamental na fisiologia das ilhotas pancreáticas, e variações genéticas neste fator de transcrição causam defeito funcional nas células $\beta$ pancreáticas, resultando em uma perda progressiva da secreção de insulina, com consequente aumento da glicemia e glicosúria. O quadro clínico do diabetes causado pela mutação nos genes HNF-4 $\alpha$ (MODY 1) e HNF-1 $\alpha$ (MODY 3) é semelhante, e são comuns as complicações microvasculares relacionadas com o controle glicêmico $(21,22)$.

A sulfoniluréia é um medicamento efetivo no controle inicial da hiperglicemia de pacientes com essa mutação, reduzindo a transcrição funcional deste fator no metabolismo da glicose, através da estimulação de insulina pela ativação de ATP e os canais de potássio $(22 ; 38)$.

\section{MODY 4}

O fator Pdx-1 (pancreatic duodenal homeobox 1), conhecido também como fator promotor de insulina (IPF-1), codifica a transcrição de regulação e desenvolvimento das células $\beta$ das ilhotas de Langerhans, a maturidade das células beta pancreáticas e o mecanismo de secreção de insulina $(8,39)$. A variação heterozigótica no IPF-1, localizado no braço longo do cromossomo 13 (13q12.1) leva a resistência a insulina. Em mutações homozigóticas, identificou-se agenesia pancreática. Acredita-se que este subtipo de MODY seja o mais raro e apresente o menor risco de apresentar complicações crônicas, quando comparado com os subtipos 1,3 e 5 (30, 39). 


\section{MODY 5}

O MODY 5 foi descrito pela primeira vez por japoneses que observaram uma expressão reduzida do fator nuclear de transcrição do hepatócito 1 beta (HNF-1 $\beta$ ) em rins de indivíduos com diabetes tipo MODY, e associaram com a disfunção renal que estes indivíduos apresentavam (40). Os carreadores da mutação podem apresentar agenesia da cabeça e corpo do pâncreas ou anomalias renais associados ao diabetes $(38,41-43)$.

O gene HNF-1 $\beta$ é importante na regulação e expressão gênica de vários órgãos incluindo pâncreas, fígado, rins e trato genito-urinário. Na mutação associada com o diabetes, geralmente há atrofia e disfunção pancreática exócrina, interferido na secreção de insulina (44).

\section{MODY 6}

O papel do gene NeuroD1/Beta2 é de promover a diferenciação neuronal e o desenvolvimento morfológico do pâncreas. Variações no fator de transcrição NeuroD1/Beta2 levam ao defeito na secreção de insulina pelas células beta pancreáticas, por mecanismos ainda não bem descritos $(38,45)$. De baixa prevalência, as variações podem causar sinais e sintomas moderados a severos (30).

\section{Outros subtipos de MODY}

Uma parcela considerável dos diabéticos fenotipicamente compatíveis com MODY não possui mutação em um dos genes MODY conhecidos (21), o que estimula o estudo de outras variações gênicas que determinem outras formas de MODY. Esses genes MODY desconhecidos têm sido denominados por alguns autores de MODY X, e podem representar de 15 a $45 \%$ dos casos de MODY, podendo chegar a $80 \%$ dependendo da população estudada $(46,47)$.

O KLF11 (do inglês, Kruppel-like fator) regula o crescimento das células exócrinas do pâncreas e atua como um supressor tumoral em tumores pancreáticos malignos; além de participar da sinalização da glicose nas células beta. Variações no gene KFL11 foram associadas com diabetes monogênico, caracterizando o MODY 7 (48).

O MODY 8 foi designado quando um estudo encontrou uma deleção no gene da carboxil-ester lipase (CEL) de indivíduos com critérios diagnósticos positivos para MODY, mas que não se enquadravam nos subtipos de MODY conhecidos. Estes indivíduos apresentavam dor abdominal e deficiência de elastase fecal, indicando o comprometimento do pâncreas. O gene CEL controla funções pancreáticas endócrinas e exócrinas (35).

$\mathrm{O}$ fator de transcrição PAX4 é fundamental para o desenvolvimento das células beta pancreáticas. Um estudo associou uma mutação no gene PAX4 com diabetes tipo MODY em pacientes com características clínicas compatíveis com MODY, mas que não possuíam as mutações conhecidas dos outros subtipos. Estava então proposto o MODY $9(49,50)$. E mais recentemente foi relatada uma mutação no gene $\mathrm{ABCC} 8$ que pode causar um tipo raro de MODY (51).

\section{CONCLUSÃO}

Por muito tempo, pacientes com diabetes eram classificados como tipo 1 ou 2 com base em critérios muito simplistas, geralmente com base na idade do paciente. $\mathrm{O}$ entendimento sobre o diabetes tem evoluído ao longo das décadas de estudo, entretanto ainda existem lacunas em relação à sua fisiopatologia, provavelmente por seu caráter multifatorial e heterogeneidade genética. Fica evidente que o correto diagnóstico e classificação do diabetes, frente às diferentes formas de apresentação e etiologia, não são tão simples e devem se basear na somatória de evidências clínicas e moleculares.

O diabetes tipo MODY é uma forma monogênica de DM2 com transmissão autossômica dominante, apresentando defeitos na secreção de insulina. As características clínicas do MODY incluem idade inferior a 45 anos no momento do diagnóstico, ausência de auto-imunidade contra as células beta pancreáticas, presença de síndrome metabólica, forte histórico familiar e produção endógena de insulina. A identificação dos genes responsáveis pela hiperglicemia auxilia no diagnóstico do MODY em indivíduos que estão sendo considerados portadores de DM2. O conhecimento destes genes pode auxiliar na classificação de crianças e jovens adultos de risco e os que poderão desenvolver a doença, podendo diminuir os riscos de hiperglicemia crônica e das co-morbidades ocasionadas pela doença.

E, por fim, a despeito do custo das análises moleculares e dificuldade de acesso por parte de algumas populações, os avanços no diagnóstico molecular do DM podem possibilitar tratamentos mais efetivos pela aplicação da farmacogenética, além de permitir um prognóstico familiar mais adequado. 


\section{REFERÊNCIAS}

1. Yamagishi S, Maeda S, Matsui T, Ueda S, Fukami K, Okuda S Role of advanced glycation end products (AGEs) and oxidative stress in vascular complications in diabetes. Biochem Biophys Acta. 2012;1820(5):663-71

2. Tsang MW. The management of type 2 diabetic patients with hypoglycaemic agents. ISRN Endocrinol. 2012;2012:478120.

3. Gross JL, Silveiro SP, Camargo JL, Reichelt AJ, Azevedo MJ. Diabetes Melito: Diagnóstico, Classificação e Avaliação do Controle Glicêmico. Arq Bras Endocrinol Metab 2002;46(1):16-26.

4. ADA. Diagnosis and classification of diabetes mellitus. Diabetes Care. 2013;36 Suppl 1:S67-74.

5. van Dieren S, Beulens JW, van der Schouw YT, Grobbee DE, Neal B. The global burden of diabetes and its complications: an emerging pandemic. European journal of cardiovascular prevention and rehabilitation Official J Eur Soc Cardiol, Working Groups on Epidemiology \& Prevention and Cardiac Rehabilitation and Exercise Physiology. 2010;17 Suppl 1:S3-8

6. BRASIL. Portal da Saúde - Diabetes. Ministério da Saúde; 2013 [cited 201214 set 2012]; Available from: http://portalsaude.saude.gov.br// portalsaude/arquivos/pdf/2012/Mai/09/Vigitel_2011_ diabetes final.pdf.

7. Rubio Cabezas O, Argente J. Diabetes mellitus: clinical presentation and differential diagnosis of hyperglycemia in childhood and adolescence. An Pediatr (Barc). 2012;77(5):344 e1- e16.

8. Della Manna T. Not every diabetic child has type 1 diabetes mellitus. J Pediatria. 2007;83(5 Suppl):S178-83.

9. Lawrence JM, Mayer-Davis EJ, Reynolds K, Beyer J, Pettitt DJ D'Agostino RB, Jr., et al. Diabetes in Hispanic American youth: prevalence, incidence, demographics, and clinical characteristics: the SEARCH for Diabetes in Youth Study. Diabetes Care. 2009;32 Suppl 2:S123-32.

10. Rewers M. Challenges in diagnosing type 1 diabetes in different populations. Diabetes \& Metabol J. 2012;36(2):90-7.

11. Keller N, Bhatia S, Braden JN, Gildengorin G, Johnson J, Yedlin $\mathrm{R}$, et al. Distinguishing type 2 diabetes from type 1 diabetes in African American and Hispanic American pediatric patients. Plos One. 2012;7(3):e32773

12. Ramesh SC, Marshall I. Clinical suspicion of Maturity Onset of Diabetes of the Young in pediatric patients diagnosed with diabetes mellitus. Ind J Pediatrics. 2012;79(7):955-8.

13. Fajans SS, Bell GI, Polonsky KS. Molecular mechanisms and clinical pathophysiology of maturity-onset diabetes of the young. New Engl J Med. 2001;345(13):971-80.

14. Gardner DS, Tai ES. Clinical features and treatment of maturity onset diabetes of the young (MODY). Diabetes, metabolic syndrome and obesity : targets and therapy. 2012;5:101-8.

15. Timshina DK, Thappa DM, Agrawal A. A clinical study of dermatoses in diabetes to establish its markers. Indian journal of dermatology. 2012;57(1):20-5.

16. Barbato MT, Criado PR, Silva AK, Averbeck E, Guerine MB, Sa NB. Association of acanthosis nigricans and skin tags with insulin resistance. Anais Bras Dermatol. 2012;87(1):97-104.
17. Schober E, Rami B, Grabert M, Thon A, Kapellen T, Reinehr $\mathrm{T}$, et al. Phenotypical aspects of maturity-onset diabetes of the young (MODY diabetes) in comparison with Type 2 diabetes mellitus (T2DM) in children and adolescents: experience from a large multicentre database. Diabetic medicine : a journal of the British Diabetic Association. 2009;26(5):466-73.

18. Fajans SS, Conn JW. Tolbutamide-induced improvement in carbohydrate tolerance of young people with mild diabetes mellitus. Diabetes. 1960;9:83-8.

19. Velho G, Froguel P. Genetic, metabolic and clinical characteristics of maturity onset diabetes of the young. Eur J Endocrinol/ Eur Fed Endocrine Soc. 1998;138(3):233-9.

20. Klupa T, Warram JH, Antonellis A, Pezzolesi M, Nam M, Malecki MT, et al. Determinants of the development of diabetes (maturity-onset diabetes of the young-3) in carriers of HNF-1alpha mutations: evidence for parent-of-origin effect. Diabetes Care. 2002;25(12):2292-301.

21. Naylor R, Philipson LH. Who should have genetic testing for maturity-onset diabetes of the young? Clinical Endocrinol. 2011;75(4):422-6.

22. Oliveira CSV, Furuzawa GK, Reis AF. Diabetes mellitus tipo MODY. Arq Bras Endocrinol Metab. 2002;42(2):186-92.

23. Barrio R, Bellanne-Chantelot C, Moreno JC, Morel V, Calle $\mathrm{H}$, Alonso $\mathrm{M}$, et al. Nine novel mutations in maturity-onset diabetes of the young (MODY) candidate genes in 22 Spanish families. The Journal of clinical endocrinology and metabolism. 2002;87(6):2532-9.

24. Ellard S, Bellanne-Chantelot C, Hattersley AT. Best practice guidelines for the molecular genetic diagnosis of maturity-onset diabetes of the young. Diabetologia. 2008;51(4):546-53.

25. Kavvoura FK, Owen KR. Maturity onset diabetes of the young: clinical characteristics, diagnosis and management. Pediatric endocrinology reviews : PER. 2012;10(2):234-42.

26. Owen KR. Monogenic diabetes: old and new approaches to diagnosis. Clin Med. 2013;13(3):278-81.

27. Gloyn AL, Faber JH, Malmodin D, Thanabalasingham G, Lam F, Ueland PM, et al. Metabolic profiling in Maturity-onset diabetes of the young (MODY) and young onset type 2 diabetes fails to detect robust urinary biomarkers. Plos One. 2012;7(7):e40962.

28. Mughal SA, Park R, Nowak N, Gloyn AL, Karpe F, Matile H, et al. Apolipoprotein M can discriminate HNF1A-MODY from Type 1 diabetes. Diabetic medicine : a journal of the British Diabetic Association. 2013;30(2):246-50.

29. Stanger BZ. HNF4A and diabetes: injury before insult? Diabetes. 2008;57(6):1461-2.

30. Sánchez-Reyes L, Fanghänel G, Márquez-Cid ME, Rocha RS, Labastida-Sánchez C, Solís-Pérez A, et al. Actualización em los diferentes subtipos diabetes tipo "MODY". Rev Endocrinología y Nutrición. 2001;9(1):5-11.

31. Holmkvist J, Almgren P, Lyssenko V, Lindgren CM, Eriksson $\mathrm{KF}$, Isomaa $\mathrm{B}$, et al. Common variants in maturity-onset diabetes of the young genes and future risk of type 2 diabetes. Diabetes. 2008;57(6):1738-44 
32. Pearson ER, Pruhova S, Tack CJ, Johansen A, Castleden HA, Lumb PJ, et al. Molecular genetics and phenotypic characteristics of MODY caused by hepatocyte nuclear factor 4alpha mutations in a large European collection. Diabetologia. 2005;48(5):878-85.

33. Martin D, Bellanne-Chantelot C, Deschamps I, Froguel P, Robert JJ, Velho G. Long-term follow-up of oral glucose tolerance testderived glucose tolerance and insulin secretion and insulin sensitivity indexes in subjects with glucokinase mutations (MODY2). Diabetes Care. 2008;31(7):1321-3.

34. Mohan V. Type 2 diabetes can also be multigenerational like MODY. Int J Diabetes Dev Ctries. 2011;31(3):125-7.

35. Nyunt O, Wu JY, McGown IN, Harris M, Huynh T, Leong GM, et al. Investigating maturity onset diabetes of the young. The Clinical biochemist Reviews / Australian Association of Clinical Biochemists. 2009;30(2):67-74.

36. Noorian S, Sayarifard F, Farhadi E, Barbetti F, Rezaei N. GCK Mutation in a Child with Maturity Onset Diabetes of the Young, Type 2. Iranian journal of pediatrics. 2013;23(2):226-8.

37. Velho G, Vaxillaire M, Boccio V, Charpentier G, Froguel P. Diabetes complications in NIDDM kindreds linked to the MODY3 locus on chromosome 12q. Diabetes Care. 1996;19(9):915-9.

38. Murphy R, Ellard S, Hattersley AT. Clinical implications of a molecular genetic classification of monogenic beta-cell diabetes. Nature clinical practice Endocrinology \& metabolism. 2008;4(4):200-13.

39. Sachdeva MM, Claiborn KC, Khoo C, Yang J, Groff DN, Mirmira RG, et al. Pdx1 (MODY4) regulates pancreatic beta cell susceptibility to ER stress. Proc Natl Acad Sci U S A. 2009; 106(45):19090-5.

40. Horikawa Y, Iwasaki N, Hara M, Furuta H, Hinokio Y, Cockburn $\mathrm{BN}$, et al. Mutation in hepatocyte nuclear factor-1 beta gene (TCF2) associated with MODY. Nature genetics. 1997;17(4):384-5.

41. Bellanne-Chantelot C, Chauveau D, Gautier JF, Dubois-Laforgue D, Clauin S, Beaufils S, et al. Clinical spectrum associated with hepatocyte nuclear factor-1beta mutations. Annals of internal medicine. 2004;140(7):510-7.
42. Ulinski T, Lescure S, Beaufils S, Guigonis V, Decramer S, Morin $\mathrm{D}$, et al. Renal phenotypes related to hepatocyte nuclear factor1 beta (TCF2) mutations in a pediatric cohort. J Am Soc Nephrol. 2006;17(2):497-503.

43. Haldorsen IS, Vesterhus M, Raeder H, Jensen DK, Sovik O, Molven A, et al. Lack of pancreatic body and tail in HNF1B mutation carriers. Diabetic medicine : a journal of the British Diabetic Association. 2008;25(7):782-7.

44. Edghill EL, Oram RA, Owens M, Stals KL, Harries LW, Hattersley AT, et al. Hepatocyte nuclear factor-1beta gene deletions--a common cause of renal disease. Nephrology, dialysis, transplantation : official publication of the European Dialysis and Transplant Association - European Renal Association. 2008;23(2):627-35.

45. Chu K, Tsai MJ. Neuronatin, a downstream target of BETA2/ NeuroD1 in the pancreas, is involved in glucose-mediated insulin secretion. Diabetes. 2005;54(4):1064-73.

46. Pruhova S, Ek J, Lebl J, Sumnik Z, Saudek F, Andel M, et al. Genetic epidemiology of MODY in the Czech republic: new mutations in the MODY genes HNF-4alpha, GCK and HNF-1alpha. Diabetologia. 2003;46(2):291-5.

47. Xu JY, Dan QH, Chan V, Wat NM, Tam S, Tiu SC, et al. Genetic and clinical characteristics of maturity-onset diabetes of the young in Chinese patients. European journal of human genetics : EJHG. 2005;13(4):422-7.

48. Neve B, Fernandez-Zapico ME, Ashkenazi-Katalan V, Dina C, Hamid YH, Joly E, et al. Role of transcription factor KLF11 and its diabetes-associated gene variants in pancreatic beta cell function. Proc Natl Acad Sci U S A. 2005;102(13):4807-12.

49. Plengvidhya N, Kooptiwut S, Songtawee N, Doi A, Furuta H, Nishi M, et al. PAX4 mutations in Thais with maturity onset diabetes of the young. The Journal of clinical endocrinology and metabolism. 2007;92(7):2821-6.

50. Jo W, Endo M, Ishizu K, Nakamura A, Tajima T. A novel PAX4 mutation in a Japanese patient with maturity-onset diabetes of the young. Tohoku J Exp Med. 2011;223(2):113-8.

51. Hartemann-Heurtier A, Simon A, Bellanne-Chantelot C, Reynaud R, Cave H, Polak M, et al. Mutations in the ABCC8 gene can cause autoantibody-negative insulin-dependent diabetes. Diabetes \& metabolism. 2009;35(3):233-5. 